\title{
Intraoperative near-infrared spectroscopy for pedicled perforator flaps: a possible tool for the early detection of vascular issues
}

\author{
Andrea Marchesi, Pietro Garieri, Francesco Amendola, Stefano Marcelli, Luca Vaienti \\ Department of Plastic Surgery, Hand Surgery and Reconstructive Microsurgery, Ospedale San Gerardo, Monza, Italy
}

Background Pedicled perforator flaps can present postoperative complications similar to those encountered in free flap surgery. Beyond a clinical evaluation, there is still no reliable technical aid for the early prediction of vascular issues. The aim of this study was to assess the support of near-infrared spectroscopy technology as an intraoperative tool to anticipate postsurgical flap ischemia.

Methods We prospectively enrolled 13 consecutive patients who were referred to our hospital from March 2017 to July 2018 and required a reconstructive procedure with a pedicled fasciocutaneous perforator flap. We measured flap peripheral capillary oxygen saturation $\left(\mathrm{SpO}_{2}\right)$ in each patient with a Somanetics INVOS 5100C Cerebral/Somatic Oximeter (Medtronic), both before and after transposition. Patient demographics, operative data, and complications were then recorded during the following 6 months. We analyzed the data using the Wilcoxon signed-rank test and linear regression.

Results The mean flap $\mathrm{SpO}_{2}$ before and after transposition was $92 \% \pm 3 \%$ and $78 \% \pm 19 \%$, respectively. The mean change in $\mathrm{SpO}_{2}$ was $14 \% \pm 17 \%$, with a range of $0 \%$ to $55 \%$. The change in saturation and mean saturation ratio were significantly different between patients with and without postoperative flap necrosis.

Conclusions An immediate quantitative analysis of flap peripheral capillary $\mathrm{SpO}_{2}$ after transposition has never before been described. In our experience, an intraoperative drop in $\mathrm{SpO}_{2}$ equal to or greater than 15\%-20\% predicted vascular complications in pedicled perforator flaps. Conversely, flap size and rotation angle were not correlated with the risk of flap necrosis.

Keywords Perforator flap / Oximetry / Monitoring / Surgical flap / Postoperative complications
Correspondence: Andrea Marchesi Department of Plastic Surgery, Hand Surgery and Reconstructive Microsurgery, Ospedale San Gerardo, Via Pergolesi 33, Monza 20900, Italy Tel: $+39-39-2332578$

Fax: +39-2-52774334

E-mail: ilmarchesiandrea@gmail.com

Received: March 9, 2019 • Revised: April 28, 2021 • Accepted: May 21, 2021

pISSN: 2234-6163 • elSSN: 2234-6171 • https://doi.org/10.5999/aps.2019.00311 • Arch Plast Surg 2021;48:457-461

\section{INTRODUCTION}

Perforator flaps are a reliable reconstructive technique, with versatility and minimal donor site morbidity [1-3]. High mobility and ample transposition are clear advantages of perforator flap surgery. However, flap movement directly affects the small perforator pedicles. When used as pedicled flaps, venous congestion and partial necrosis have been reported to occur in up to 
$42 \%$ of cases $[4,5]$

The early detection of possible complications is related to the surgeon's experience, and is essentially based on flap clinical monitoring, including skin color, turgor, swelling, capillary refill time, temperature, and bleeding characteristics after a pinprick [6-8]. All of these evaluations are made postoperatively, when little can be changed in the planned reconstruction.

Many new instrumental techniques have been proposed to support clinical monitoring of free flaps $[9,10]$. Near-infrared spectroscopy (NIRS)-based technology measures the capillary blood oxygen saturation $\left(\mathrm{SpO}_{2}\right)$ in the dermal capillaries, immediately after its noninvasive application. A sterile probe can be used for intraoperative measurements. However, the use of NIRS in flap monitoring has only been reported in two animal studies. A decrease in $\mathrm{SpO}_{2}$ within hours after flap elevation was reported to predict flap viability $[11,12]$.

The aim of this study was to use NIRS technology for the immediate evaluation of flap vascularization and to assess the ability of immediate NIRS to predict the postoperative clinical course.

\section{METHODS}

We prospectively analyzed 12 consecutive patients needing 13 pedicled perforator flaps for reconstructive surgery, who were referred to our department from March 2017 to July 2018. All patients were preoperatively studied with Huntleigh Doppler ultrasound for perforator vessel identification and mapping. Intraoperative flap superficial oximetry was recorded with Somanetics INVOS 5100C Cerebral/Somatic Oximeter (Medtronic,

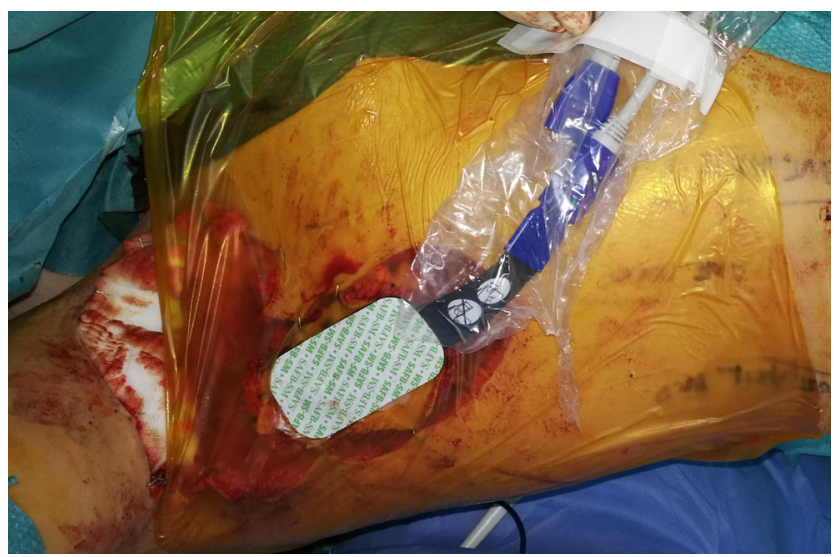

Fig. 1. A patient who suffered from Hurley stage III hidradenitis suppurativa and was treated with wide local excision followed by immediate reconstruction with a thoracodorsal artery perforator (TDAP) flap. In the case shown here, we intraoperatively monitored the pedicled TDAP flap with near-infrared spectroscopy before its transposition into the axillary defect.
Dublin, Ireland) sterile patch sensors, both before transposition and after transposition of the flap to the definitive recipient site (Figs. 1, 2).

The change in $\mathrm{SpO}_{2}\left(\Delta \mathrm{SpO}_{2}\right.$, expressed in percentage points) indicates the absolute intraoperative drop in $\mathrm{SpO}_{2}$. The $\mathrm{SpO}_{2}$ ratio, defined as $\left(\mathrm{SpO}_{2}\right.$ before transposition-SpO 2 after transposition) $/ \mathrm{SpO}_{2}$ before transposition, refers to the relative intraoperative drop of $\mathrm{SpO}_{2}$ and allows a better comparison of results, independent from the absolute values of $\mathrm{SpO}_{2}$. The arc of rotation (in degrees) and area of harvesting (in square centimeter) were registered intraoperatively for every pedicled flap. In all cases, perforator identification, oximetry measurement, and flap dissection were performed by the same surgeon in the same department. The differences between oximetry changes were evaluated using the Wilcoxon signed-rank test. The association between flap saturation and necrosis occurrence was analyzed using linear regression analysis.

\section{RESULTS}

Seven male and five female patients were referred to our department for reconstruction with a perforator flap. Nine patients suffered from axillary Hurley stage III hidradenitis suppurativa. A thoraco-dorsal artery perforator flap was the most frequent reconstruction. Patients' mean age was $38.7 \pm 20.0$ years, with a mean body mass index (BMI) of $26.5 \pm 5.1 \mathrm{~kg} / \mathrm{m}^{2}$. Seven patients were active smokers. Only one patient had diabetes mellitus. No other known comorbidities were present that would constitute a risk factor for the outcomes of perforator flap reconstruction. Details about the diseases and reconstruction tech-

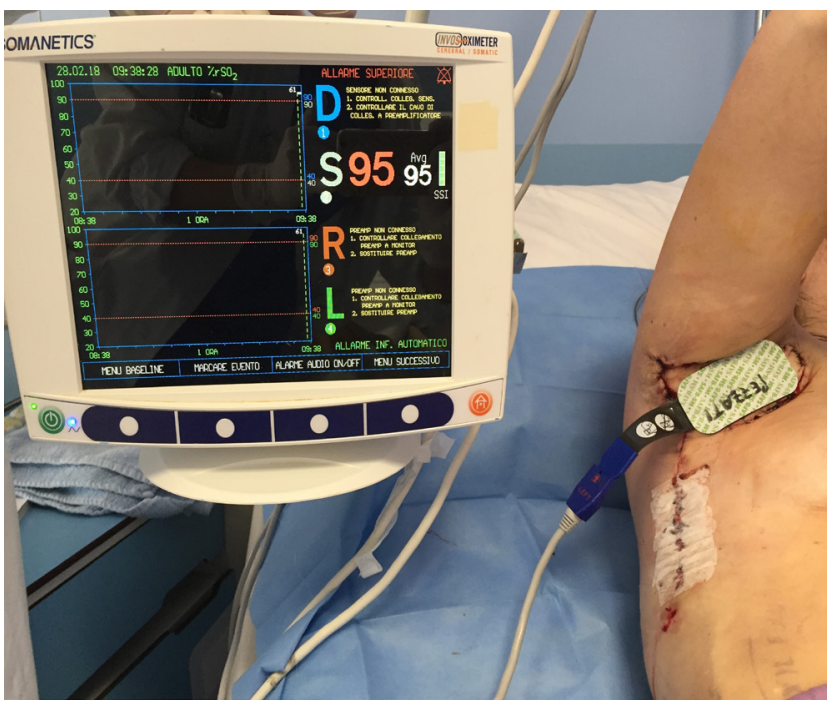

Fig. 2. Postoperative monitoring of a pedicled thoracodorsal artery perforator flap with near-infrared spectroscopy after flap inset. 
niques are outlined in Table 1.

The mean flap area was $82.79 \pm 32.88 \mathrm{~cm}^{2}$, with a mean rotation angle of $114.17^{\circ} \pm 35.02^{\circ}$. The mean flap $\mathrm{SpO}_{2}$ before and after transposition were $92 \% \pm 3 \%$ and $78 \% \pm 19 \%$, respectively. The mean $\Delta \mathrm{SpO}_{2}$ registered was $14 \% \pm 17 \%$, with a range of $0 \%$ to $55 \%$. The mean $\mathrm{SpO}_{2}$ ratio was $15 \% \pm 19 \%$. Details about flap characteristics and oximetry measurements are outlined in Table 2.

Partial necrosis of the flap occurred in three patients, and total necrosis was observed in only one patient (the patient with diabetes mellitus).

No differences in age, BMI, smoking history, flap dimensions, or arc of rotation were found to be statistically significant between patients with and without postoperative flap necrosis. Linear regression analysis did not show significant associations of BMI, flap size, and rotation angle with the saturation ratio.

No significant difference was found in before-transposition flap saturation between patients with and without necrosis. However, differences in after transposition $\mathrm{SpO}_{2}$ (61.6\% vs. $89.6 \%, \mathrm{P}=0.025), \Delta \mathrm{SpO}_{2}(28.8 \%$ vs. $3.1 \%, \mathrm{P}=0.021)$ and the mean saturation ratio ( $32.3 \%$ vs $3.5 \%, \mathrm{P}=0.027)$ were found to be significant between patients with and without postoperative flap necrosis.

Despite the significant difference in the mean saturation ratio between the necrotic and non-necrotic flaps, no significant correlation was found between the saturation ratio and the percentage of necrotic area.

\section{DISCUSSION}

Perforator flap reconstruction is a transposition of skin islands, based on tiny arterial vessels usually smaller than $2 \mathrm{~mm}$. Flap movement affects the perforator pedicle, but its effects may be detectable only after some hours after the operation. Prompt detection of a vascular issue in pedicled perforator flaps may help the surgeon to reduce the complication rate. Thus, surgeons have consistently pointed out the need for technical support to early predict possible vascular complications.

In 1975, Creech and Miller outlined the criteria they considered essential for an "ideal" free flap monitoring device [13]. These included safety to the patient and flap, applicability to all flap types, rapid response to blood flow changes, accuracy, affordability, and ease of interpretation [14]. Many techniques have been proposed, such as an implantable Doppler ultrasound

Table 2. Intraoperative flap superficial oximetry values before and after transposition of the flap to the definitive recipient site

\begin{tabular}{lccccc}
\hline Patient & $\mathrm{BT} \mathrm{SpO}_{2}(\%)$ & $\mathrm{AT} \mathrm{SpO}$ & $\mathrm{SSpO}_{2}$ & $\mathrm{SpO}_{2}$ ratio & Necrosis \\
\hline 1 & 95 & 78 & 17 & 18 & 1 \\
2 & 90 & 68 & 22 & 24 & 1 \\
3 & 94 & 61 & 33 & 35 & 1 \\
3 & 88 & 71 & 17 & 19 & 1 \\
4 & 85 & 30 & 55 & 65 & 1 \\
5 & 95 & 95 & 0 & 0 & 0 \\
6 & 89 & 85 & 4 & 4 & 0 \\
7 & 88 & 79 & 9 & 10 & 0 \\
8 & 95 & 95 & 0 & 0 & 0 \\
9 & 92 & 81 & 11 & 12 & 0 \\
10 & 93 & 92 & 1 & 1 & 0 \\
11 & 95 & 95 & 0 & 0 & 0 \\
12 & 95 & 95 & 0 & 0 & 0 \\
Mean Necr & 90.4 & 61.6 & 28.8 & 32.3 & \\
Mean No Necr & 92.75 & 89.6 & 3.1 & 3.5 & \\
P-value & 0.31 & $<0.05$ & $<0.05$ & $<0.05$ & \\
\hline
\end{tabular}

$\triangle \mathrm{SpO}_{2}$ and the $\mathrm{SpO}_{2}$ ratio indicate the absolute and relative intraoperative change in $\mathrm{SpO}_{2}$.

$\mathrm{BT}$, before transposition; $\mathrm{AT}$, after transposition; $\mathrm{SpO}_{2}$, saturated oxygen; Necr, flaps with partial or total necrosis; No Necr, flaps without necrosis.

Table 1. Patient demographics and operative data

\begin{tabular}{|c|c|c|c|c|c|c|c|}
\hline Patient & Age (yr) & BMI $\left(\mathrm{kg} / \mathrm{m}^{2}\right)$ & Smoke & Diagnosis & Flap & Area $\left(\mathrm{cm}^{2}\right)$ & Rotation $\left(^{\circ}\right)$ \\
\hline 1 & 47 & 38 & + & HS & TDAP & 144 & 100 \\
\hline 2 & 54 & 21.6 & - & Trauma & PTP & 36 & 110 \\
\hline 3 & 51 & 26.6 & + & $\mathrm{HS}$ & SIEA & 90 & 90 \\
\hline 3 & 51 & 26.6 & - & HS & SIEA & 30 & 90 \\
\hline 4 & 19 & 32 & + & HS & TDAP & 98 & 100 \\
\hline 5 & 19 & 20.9 & + & HS & TDAP & 84 & 120 \\
\hline 6 & 24 & 30.3 & + & HS & TDAP & 80 & 110 \\
\hline 7 & 36 & 22 & + & Trauma & ALT & 78 & 170 \\
\hline 8 & 21 & 24.7 & + & HS & TDAP & 52.5 & 60 \\
\hline 9 & 85 & 24 & - & Skin cancer & ALT & 126 & 60 \\
\hline 10 & 52 & 30.9 & - & Skin cancer & DIEP & 90 & 90 \\
\hline 11 & 20 & 20.8 & - & $\mathrm{HS}$ & TDAP & 84 & 170 \\
\hline 12 & 24 & 25.9 & - & HS & TDAP & 84 & 100 \\
\hline Mean \pm SD & $38.69 \pm 20.01$ & $26.48 \pm 5.12$ & & & & $82.79 \pm 32.88$ & $114.17 \pm 35.02$ \\
\hline
\end{tabular}

BMI, body mass index; HS, hidradenitis suppurativa; TDAP, thoracodorsal artery perforator; PTP, posterior tibial artery perforator; SIEA, superficial inferior epigastric artery; ALT, antero-lateral thigh; DIEP, deep inferior epigastric perforator. 
probe, pulse oximetry, laser Doppler flowmetry, color Duplex sonography, NIRS, and tissue $\mathrm{pH}$ monitoring with microdialysis [15]. Although all these devices have improved flap salvage rates, none has proved to be superior to others.

NIRS is a tool for noninvasive measurement of tissue components. It is operator-independent and easy to manage, even by non-experts. It is important to avoid unstable probe contact and external light irradiation that can cause impossible or incorrect measurements. Regarding free flaps, NIRS has been reported to be a reliable technique to accurately detect decreases in flap perfusion (sensitivity, 99.1\%; specificity, 99.9\%) [14,16]. As reported in the literature, NIRS seems to detect vascular compromise of free flaps prior to clinical or Doppler monitoring $[17,18]$.

Ozturk et al. [19] demonstrated no significant correlation between $\mathrm{SpO}_{2}$ and blood pressure, supplemental $\mathrm{O}_{2}$, flap type, perforator number, or vessel caliber. There is also no universally accepted consensus regarding a risk threshold during free-flap monitoring by NIRS, but the most widely supported criteria, proposed by Keller, indicate that a flap $\mathrm{SpO}_{2}$ lower than $30 \%$ or a drop rate in $\mathrm{SpO}_{2}$ equal to or greater than $20 \%$ per hour sustained for more than 30 minutes is predictive for vascular complications [20]. In our experiences with pedicled perforator flaps, an intraoperative drop rate in $\mathrm{SpO}_{2}$ ratio equal to or greater than $15 \%-20 \%$ was significantly associated with partial or total flap necrosis. Thus, intraoperative NIRS seems to be a useful tool to predict vascular issues in the very first minutes after flap inset, when it is otherwise clinically silent. However, it should be kept in mind that the NIRS probes commercialized for flap monitoring have a measurement depth within $10 \mathrm{~mm}$ [21].

We did not find significant correlations between the saturation ratio and the percentage of necrotic area. This finding may be related to the restricted number of analyzed patients, and further studies are needed to confirm or refute our results. Finally, we did not find significant associations of BMI, flap size, and rotation angle with the saturation ratio. These data seem to suggest that pedicled perforator flaps are equally safe in different patients and defects.

The average before-transposition $\mathrm{SpO}_{2}$ was overall good, ranging from $85 \%$ to $95 \%$. Therefore, these perforator vessels were consistently sufficient to perfuse the entire skin paddle. In our case series, necrosis was most likely related to an insufficient length of the dissected pedicles to tolerate the torsion or minimal, but significant kinking. The ideal vessels should be larger than $0.5 \mathrm{~mm}$ in caliber and pulsatile, and the pedicle length should be at least $4 \mathrm{~cm}$ to reduce the risk of torsion.

An intraoperative drop rate in $\mathrm{SpO}_{2}$ ratio equal or greater than $15 \%-20 \%$ was predictive for vascular complications in pedicled perforator flaps. Conversely, flap size and rotation angle did not seem to be correlated with the risk of flap necrosis. Prompt detection of a vascular issue in pedicled perforator flaps could help the surgeon to reduce the complication rate; consequently, an immediate exploration of pedicle vessels is recommended to exclude the presence of kinking, twisting, or tension.

This is the first study regarding the immediate effect of flap transposition on vascularization, as measured using NIRS monitoring. The main limitations of our study are the limited population and the single-center design. Further studies are needed to analyze the efficacy of changes in the intraoperative reconstruction plan following immediate NIRS measurements.

\section{NOTES}

\section{Conflict of interest}

No potential conflict of interest relevant to this article was reported.

\section{Ethical approval}

An official institutional review board (IRB) waiver of ethical approval was obtained from the IRB of Ospedale San Raffaele (exemption No. CE-11/int/2017) and performed in accordance with the principles of the Declaration of Helsinki. Written informed consent was obtained.

\section{Patient consent}

The patients provided written informed consent for the publication and the use of their images.

\section{Author contribution}

Conceptualization: A Marchesi. Data curation: F Amendola. Formal analysis: F Amendola. Methodology: S Marcelli. Project administration: L Vaienti. Visualization: P Garieri. Writing original draft: A Marchesi, F Amendola. Writing - review \& editing: A Marchesi, F Amendola.

\section{ORCID}

Andrea Marchesi https://orcid.org/0000-0003-0493-9835

Pietro Garieri https://orcid.org/0000-0002-7929-1802

Francesco Amendola https://orcid.org/0000-0002-1410-7568

Stefano Marcelli https://orcid.org/0000-0002-2066-9460

Luca Vaienti https://orcid.org/0000-0002-5309-5205

\section{REFERENCES}

1. Marchesi A, Marcelli S, Zingaretti N, et al. Pedicled thoracodorsal artery perforator and muscle-sparing latissimus dorsi flaps in the axillary reconstruction after hidradenitis 
suppurativa excision: functional and aesthetic issues. Ann Plast Surg 2018;81:694-701.

2. Vaienti L, Calori GM, Leone F, et al. Posterior tibial artery perforator flaps for coverage of Achilles region defects. Injury 2014;45 Suppl 6:S133-7.

3. Marchesi A, Amendola F, Bordone F, et al. Muscle electrical activity after perforator flap surgery: a pilot cohort study. Eur J Plast Surg 2020;43:165-8.

4. Sisti A, D’Aniello C, Fortezza L, et al. Propeller flaps: a literature review. In Vivo 2016;30:351-73.

5. Innocenti M, Menichini G, Baldrighi C, et al. Are there risk factors for complications of perforator-based propeller flaps for lower-extremity reconstruction? Clin Orthop Relat Res 2014;472:2276-86.

6. Bui DT, Cordeiro PG, Hu QY, et al. Free flap reexploration: indications, treatment, and outcomes in 1193 free flaps. Plast Reconstr Surg 2007;119:2092-100.

7. Chen KT, Mardini S, Chuang DC, et al. Timing of presentation of the first signs of vascular compromise dictates the salvage outcome of free flap transfers. Plast Reconstr Surg 2007;120:187-95.

8. Cusano A, Fernandes R. Technology in microvascular surgery. Oral Maxillofac Surg Clin North Am 2010;22:73-90.

9. Lineaweaver W. Techniques of monitoring buried fasciocutaneous free flaps. Plast Reconstr Surg 2009;124:1729-31.

10. Schmulder A, Gur E, Zaretski A. Eight-year experience of the Cook-Swartz Doppler in free-flap operations: microsurgical and reexploration results with regard to a wide spectrum of surgeries. Microsurgery 2011;31:1-6.

11. Stranc MF, Sowa MG, Abdulrauf B, et al. Assessment of tissue viability using near-infrared spectroscopy. Br J Plast Surg 1998;51:210-7.

12. Zhu C, Chen S, Chui $\mathrm{CH}$, et al. Early prediction of skin viability using visible diffuse reflectance spectroscopy and autofluorescence spectroscopy. Plast Reconstr Surg 2014;134:
$240 \mathrm{e}-247 \mathrm{e}$

13. Whitaker IS, Rozen WM, Chubb D, et al. Postoperative monitoring of free flaps in autologous breast reconstruction: a multicenter comparison of 398 flaps using clinical monitoring, microdialysis, and the implantable Doppler probe. J Reconstr Microsurg 2010;26:409-16.

14. Smit JM, Zeebregts CJ, Acosta R, et al. Advancements in free flap monitoring in the last decade: a critical review. Plast Reconstr Surg 2010;125:177-85.

15. Salgado CJ, Moran SL, Mardini S. Flap monitoring and patient management. Plast Reconstr Surg 2009;124(6 Suppl): e295-302.

16. Moubayed SP, Mourad M, Urken ML. What are the optimal monitoring techniques in head and neck microvascular reconstruction? ORL J Otorhinolaryngol Relat Spec 2016;78: 241-4.

17. Salgarello M, Pagliara D, Rossi M, et al. Postoperative monitoring of free DIEP flap in breast reconstruction with nearinfrared spectroscopy: variables affecting the regional oxygen saturation. J Reconstr Microsurg 2018;34:383-8.

18. Lohman RF, Langevin CJ, Bozkurt M, et al. A prospective analysis of free flap monitoring techniques: physical examination, external Doppler, implantable Doppler, and tissue oximetry. J Reconstr Microsurg 2013;29:51-6.

19. Ozturk CN, Ozturk C, Ledinh W, et al. Variables affecting postoperative tissue perfusion monitoring in free flap breast reconstruction. Microsurgery 2015;35:123-8.

20. Keller A. A new diagnostic algorithm for early prediction of vascular compromise in 208 microsurgical flaps using tissue oxygen saturation measurements. Ann Plast Surg 2009;62: 538-43.

21. Kagaya Y, Miyamoto S. A systematic review of near-infrared spectroscopy in flap monitoring: current basic and clinical evidence and prospects. J Plast Reconstr Aesthet Surg 2018; $71: 246-57$. 